\title{
The Analyses and Countermeasures on the Major Technology Defects of the Shell Plastic Parts
}

\author{
Zhihong Yu \\ Henan Mechanical and Electrical Engineering College, Xinxiang, Henan, P.R. China 453000 \\ Llyuxinhui@126.com
}

Keywords: Shell plastic parts, Plastic material, Injection molding process, Mould design and manufacture

\begin{abstract}
The quality of the shell plastic parts, whose production is mainly affected by the plastic material, the mould design and manufacture and the process parameters of the injection molding, has effect on the market competition of the products packaged directly. So, it is necessary to analyze the reasons and measures of the main process defects of the shell plastic parts.
\end{abstract}

\section{Introduction}

The shell plastic parts are the major structural parts involved in these industry such as home electronics products, civilian industry products and so on, whose texture, color, shape and the service life are important propaganda pages in brochure. However, various kinds of process defects such as insufficient fill, much silver, severe buckling deformation during the mass, repetitive production cause the low production quality and the high production cost, and then the comprehensive benefits and market competition of the enterprise are impacted hugely. Competitive elements such as quality and cost, relate to enterprise's core competitive ability and competitive advantage, which means it is better having high quality under the established cost or the low cost under the same quality.[1]In order to improve enterprises' competition strength by controlling the production quality and cost of shell plastic parts, a table frame of home appliance products, for example a shell plastic part, is taken as the carrier in this paper, shown in figure 1.1. Its common reasons of defects are analyzed and finishing solutions are arranged to act as a reference for the similar products to guide the manufacturing fast and accurately.

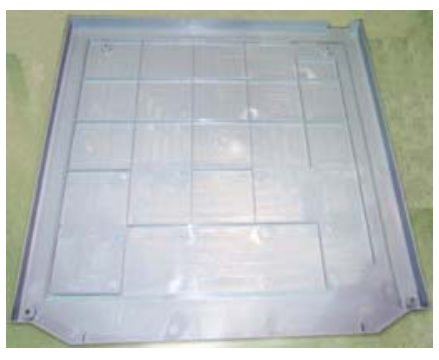

(a) the inner surface of the table frame

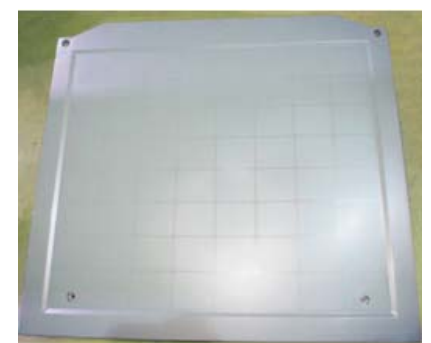

(b) the outer surface of the table frame

Fig.1 table frame

\section{Process defects and solutions of shell plastic parts in production}

When the product is small, the structure is reasonable and equipment specification meets the requirements, some usual defects of table frame during the injection mold filling stage are as follows:

Underfill. Underfill is the phenomenon that the end of material flowing is incomplete or a part of multi-cavity mold is filled in discontent. It often appears underfill in the corner as a no-gyrating parts with big size, which shown in figure 1.2. 


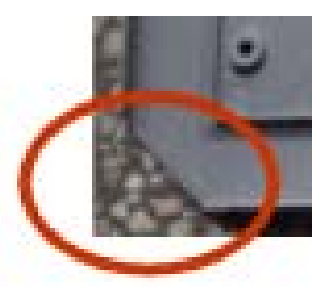

Fig.2 underfill

The main causes of the defects and the solving measures are as follows:

(1)The liquidity of Material is not good and the flow viscosity is high, so the fluid is not easy to fill the cavity. The solving measure is to choose plastic of good liquidity.

(2)Bad Exhausting of die causes so high pressure in cavity end that cavity can't be filled fully. So some air-out arks may be set up in the parting surface, as shown in figure 1.3.

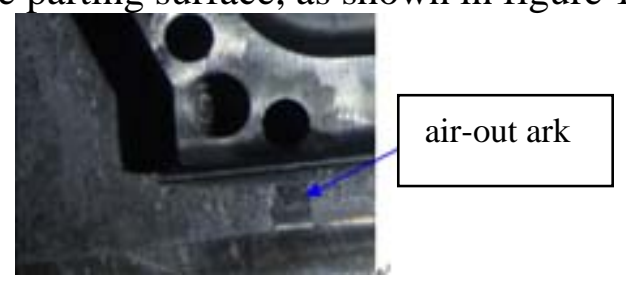

Fig.3 air-out ark

(3)Mould gating system is unreasonable, If gate location are unreasonable, gate size and runner size are too small, melt fluid will not fill cavity for impeded flow. So the design of the mould gating system should be improved. The plastic parts contour is not a body of revolution, the secondary runner , as shown in figure 1.4, is often added to increase the runner size to flow better to the four corners and to balance internal stress to ensure better full at the same time, especially for thin wall plastic parts.

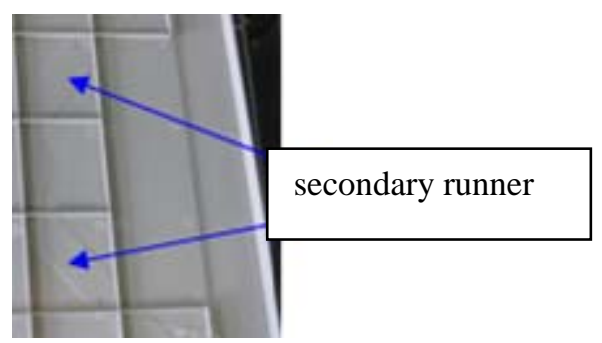

Fig.4 secondary runner

(4)Unreasonable molding process parameters such as low temperature, pressure and speed shorten the length of filling mold and cause insufficient feed. So these parameters should be increased appropriately. But the injection pressure can not be too high, otherwise buckling deformation of plastic parts will be caused because of excessive residual stress after cooling.

In addition, many other reasons have influence on filling insufficient in the production, such as much feeder, small slag hole, low nozzle temperature and short injection stroke and so on.

Sag. Sag is refers to the inward shallow pit or lacuna for inconsistency contractions in the product producing irregular surfaces of plastic parts. This plastic part often appears sag in the gate nearby, as shown in figure 1.5 .

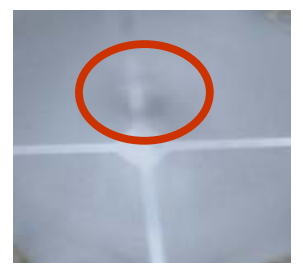

Fig.5 sag

By analyzing, the main reasons of the defects and the solving measures are as follows: 
(1)Unreasonable selection of material, For example high shrinkage ratio of raw material or bad liquidity, cause shrinkage can't be caught up in the cooling process with same pressure, which results in obvious subsidence on products surface, or high hygroscopicity that causes material flow is blocked by gas produced after melting and can not well contact with mould table wall so that sink mark appears. So it is better to choose raw materials of lower shrinkage, better fluidity and fully drying. [2][3]

(2)Bad exhausting of gas cause air entrapped, influencing melt filling into the mould, which cause sag. So the exhausting gas slot may be opened in parting surface.

(3)Unreasonable design of mould gating system. If gate's location is unreasonable and sectional area of runner is too small, sag is easy to occur for increasing resistance force of filling. So the design of the gating system is improved reasonably to solve it. For example, the gate sectional area in geometric center of the plastic parts may be expanded to improve the gating system design.

(4)Unreasonable molding process parameters often cause surface sag, such as too high melt temperature, too low pressure of the injection and short pressure retaining, and insufficient cooling of plastic part. So these parameters should be made better, for example the cooling is improved fully and uniformly.

In addition, there are other factors influencing the production of depression, for example reinforcement is not reasonable and molten material is in short supply and so on.

Silver. Silvers are these fine lines that are very long like needle and white like frost appearing plastic parts surface. This plastic part appears the defect of the silver in production, as shown in figure 6

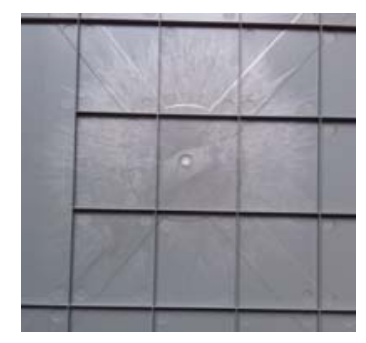

Fig.6 silver

By analyzing, the main reasons of the defects and the solving measures are as follows:

(1)Selected materials are not good, such as thermal stability of the raw material is poor, inadequate drying and high volatile matter content, they will produce a large number of gas to occur silver after heated. So choosing materials of good thermal stability or adding heat stabilizer in the material are useful.[3]

(2)Exhaust is bad. If the gas of the cavity can't be discharged as soon as possible, silver will occur. Exhaust slot will be added in the parting surface.

(3)Gating system is not reasonable, the sectional area of the passage is too small, the melt degradation will accelerate to generate silver more likely. Measure is to enlarge the runner sectional area.

(4)Molding process parameters is not reasonable, such as too high melt temperature, quick injection speed and increasing temperature, plastic will occur degradation to produce gas;[4] if the dwell time is too long , silver will occur because of gas produced accumulating; if mold temperature is too low and the cooling speed too fast, the melt will form the shell on the cavity wall when it still be filling, and then shell will be changed into blanched and muddy under all kinds of forces. So process parameters should be adjusted and used reasonably.

In addition, there are many other elements of forming silver, for example the using of the release agent is inappropriate and plasticizing uneven etc.

Fash. Fash is the phenomenon that resin overflows from parting surface of mold or clearance of accessories. The flash occurs in this plastic part, as shown in figure 7. 


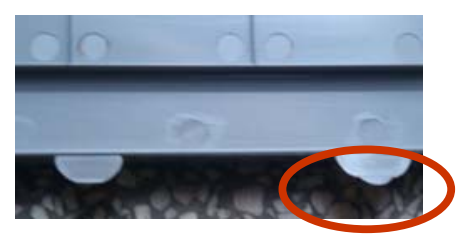

Fig.7 fash

By analyzing, the main causes of the defects and the solving measures are as follows:

(1)Material selection is not appropriate. For example, it is easy to overflow with good liquidity. Solving measure is to use higher viscosity plastic.

(2)The accuracy of manufacturing is not high or stiffness of mold material is not enough leads to greater split clearance than the maximum of not flash clearance of the plastic processed. So we should improve manufacturing accuracy of mould, or use more excellent hard steels.

(3)Molding process parameters are not reasonable. For example injection temperature or pressure is too high, dwell time is too long and injection speed is too quickly and so on. Measures are to reduce the injection temperature, pressure and speed and to improve the clamping force, etc.

In addition, there are many other factors of forming flash, for example accessories wear, mold surface attached by foreign body and shortage of clamping force.

Warping. Warping deformation refers to the phenomenon that flat places of plastic parts have ups and downs and two heads appear cocking with straight edges bending and distorting in or out.

It is concluded that the main reasons of the defects and the solving measures are as follows:

(1)Material selection is not appropriate. Crystallized resin(PE、PP、PA、POM)is more likely to warp than non-crystallized resin(PS、PC、ABS、PMMA). Under using and forming performance, non-crystallized type resin should be chosen as far as possible. [5]

(2)Design of the mould is not reasonable. If the type or position of the gate is not selected reasonably, orientation and uneven of melt flow will cause warping easily; if cooling system is not designed reasonably, cooling uneven and not fully of plastic parts will cause warping; If draft is not enough, or ejecting rods is not set reasonably, ejecting force will be too high or uneven, which cause warping. The mould should be designed reasonably. [6]

(3)Injection parameters are not reasonable. If the melt temperature is too high, the plastic parts will leads to warp due to the large internal residual stress in the quenching process; if the injection pressure or dwell pressure is too high, and the dwell time too long, the plastic parts warping will occur because of the uneven distribution of internal residual stress; if cooling time is too short, the rigid of plastic parts will be insufficient to occur warping; if melt flow rate is relatively slow, the bigger flow temperature difference is easy to produce warping. So appropriately reducing the melt temperature and injection pressure or increasing the melt flow rate, prolonging cooling time will be the correct action.

Of course, varieties of other defects such as overdo, bubble, burning and weld may appear in the injection molding process, and there are many reasons for these defects and it needs all-round analysis for specific defect. Various defects of table frame may exist in the same kind of plastic shell products, which have much influence on the internal and exterior quality of products.

\section{Conclusion}

In the fierce competition industry, we hope appearance kind of plastic parts will have high efficiency production, the above defects produced frequently fatally affect the quality of such products, and to reduce or bridge these defects is very difficult. By the above analysis, we can conclude that although all kinds of the reasons of the flaw are multidimensional and comprehensive, but there are rules, they are all because of improper material selecting, unreasonable mould design and manufacture, unreasonable molding process parameter selection, especially the design of exhaust and gating system.[7] It can help guide the production process by following the rule of defects, so as to reduce the generation of defects as far as possible, realize high quality and high efficiency production of the appearance kind of plastic parts. First of all, reasonable analysis 
forming performance of raw plastic materials combined with the product structure, guarantee it is easy for molding. Secondly, precisely design and manufacture moulds combined with product structure, guarantee casting, exhaust, good cooling and internal and external quality assurance of products.

In addition, the reasonable process parameters in the forming process is indispensable, combined with the product structure and experience to establish reasonable molding process parameters can further ensure the quality of products internal and external.

\section{References}

[1] Y.Guo:Economic Forum Vol.473 (2010), No.1 P.158 (In Chinese)

[2] H.J.Yao: Die and Mould Technology (2006), No.2 P.72 (In Chinese)

[3] F.X.Yang: Plastic Vol.32 (2003), No.6 P.59 (In Chinese)

[4] Information on http://www.uggd.com/article/cl/gcsl/625.html

[5] Y.Fu: Mechanical \& Electrical Engineering Technology Vol.35 (2005), No.5 P.33 (In Chinese)

[6] X.L.Feng: Technological Development of Enterprise Vol.31 (2012), No.16 P.35 (In Chinese)

[7] G.B.Wen: Die \& Mould Industry Vol.35 (2009), No.11 p.47 (In Chinese) 